\title{
An Optimal Routing Paradigm For Flexible Flights
}

\author{
Peng Wei ${ }^{1}$, Taehoon Kim ${ }^{2}$, Steven Landry ${ }^{2}$, Dengfeng Sun $^{1}$ and Daniel DeLaurentis ${ }^{1}$
}

\begin{abstract}
This paper introduces the concepts of metroplex and flexible flight. We focus on the routing part of a complete flexible flight operation. The authors establish a network data structure based on which the optimal routing algorithm is developed for the flexible flights which fly from their original airports to destination metroplexes. Moreover, the metroplex routing algorithm is performed under the dynamic en route traffic congestion constraint, which is rarely studied in literature. We also propose an integrated model which combines the routing and scheduling according to the practical feasible air traffic control procedure. Besides the optimal metroplex routing and the scheduling, we also brief several other functional blocks in the integrated model and illustrate the data flow inside the entire system. Finally we present the simulation results which verify the positive effect of the optimal metroplex routing algorithm and show that the total delay reduction by the integrated system is considerable.
\end{abstract}

\section{INTRODUCTION}

For an air transportation system, inefficiencies are found when air traffic demand exceeds system capacity. The resulted flight delays thus increase operating costs of airlines, air traffic controllers workload, and the probability of passengers missing connecting flights. Moreover, the air traffic demand is expected to continue its rapid growth in the future. The Federal Aviation Administration (FAA) estimated in 2007 that the number of passengers is projected to increase by an average of 3\% every year until 2025 [1]. Most of the traffic demand increase will occur in metropolitan areas where there are two or more large airports. The Joint Planning and Development Office (JPDO) defines this kind of region, with a group of two or more nearby airports whose arrival and departure operations are highly interdependent, as a metroplex. The New York Metroplex (N90 TRACON), for example, consists of John F. Kennedy (JFK) airport, LaGuardia (LGA) airport, and Newark (EWR) airport, as well as several smaller airports. The study in [2] shows that the traffic in most metroplexes has increased significantly over the past years and the N90 metroplex has the heaviest traffic demand. The future traffic growth will put the current facilities under extreme pressure. Therefore reducing delays at metroplexes is a critical objective for researchers.

There are many reasons of the inefficiencies in those busy metroplex airspace, e.g., airborne traffic congestion, airports in close proximity, and limited infrastructure resource. Separation rules and environmental problems also

\footnotetext{
${ }^{1}$ P. Wei, D. Sun and D. DeLaurentis are with the School of Aeronautics and Astronautics, Purdue University, West Lafayette, IN 47907, USA \{weip, dsun, ddelaure\} a purdue.edu

${ }^{2}$ T. Kim and S. Landry are with the School of Industrial Engineering, Purdue University, West Lafayette, IN 47907, USA $\{$ tkim, slandry $\}$ e purdue.edu
}

bring down efficiencies. To treat the the airports in the same metroplex independently does not help with decreasing the inefficiencies. Resource optimization over all the runways and airports and associated separation rules within the same metroplex will improve the overall throughput and reduce the total delay.

Although the metroplex concept and its corresponding metrics have been studied by some literature works [3]-[10], there are very few of them discussing metroplex routing. However, we noticed that several rerouting algorithms on weather avoidance were developed, which can be adapted to solve the metroplex routing problem under en route traffic congestion constraint by treating congested areas as severe weather areas. In [11] Krozel et al. proposed the weatherspecific Coded Departure Routes (CDRs) for pre-departure flights. The CDRs were calculated under the prediction based on current weather. Unfortunately, sometimes the resulted CDRs may not be feasible in practical flight operations. The authors in [12] developed a mixed data structure of a grid network and a waypoint network when computing reroutes for traffic flows approaching the destination. It mainly focused on the arrival traffic weather avoidance routing and the metrics associated with such routes. Mukherjee and Hansen [13] proposed three stochastic integer programming models for managing air traffic inbound to an airport when both the airport itself and its approach routes may be under severe weather. Compared to static routing, the dynamic rerouting algorithm they developed reduced total delay cost by exploiting updated information to make better routing decisions. Taylor and Wanke [14] presented a dynamic rerouting algorithm which generates flexible and acceptable reroutes. The research invited the experienced pilots, air traffic controllers and experts to help define the metrics of reroute acceptability and then network model was built based on the acceptable flight routes from historic data.

Capozzi et al. [15] proposed a mixed integer linear programming formulation and solved the routing and scheduling problems simultaneously for the flights with metroplex destinations. This is the only research we found on metroplex routing. Several heuristics were introduced that can be applied to speed up the algorithm to find near-optimal, satisfactory solutions. However, to handle routing and scheduling at the same time introduces a high computational complexity and is not feasible in practical scenarios.

In this paper we first introduce the concepts of metroplex and flexible flight. Then we build the network data structure model based on the waypoints (navaids, arrival fixes, metering fixes, etc.) and jet routes information. Our metroplex routing algorithm which considers the en route 
traffic congestion as the constraint is proposed. The routing is followed by the Multi-center Traffic Management Advisor (McTMA) [16] scheduler solving the runway assignment problem. To study the flexible flight behavior, we develop an estimation method to find all the candidate routes to the metroplex airports for each flight in historical data. Then the integrated model combined with optimal routing and scheduling is demonstrated and other functional blocks in the entire system are briefly introduced. To our best knowledge, this is the first work that studies the metroplex routing under the en route traffic congestion constraint and can work seamlessly with the McTMA scheduler. Finally, the simulation results of the integrated model for N90 metroplex are listed which evaluate the performances of our routing algorithm and the entire integrated system.

The rest of this paper is organized as follows. The second section introduces the concepts of metroplex and flexible flights. Section III shows how the network data structure model is established for running our metroplex routing algorithm. In the fourth section, the metroplex routing algorithm under the traffic congestion constraint is presented and the runway scheduler following the routing is also introduced. In order to observe the behavior of the flexible flights, Section $\mathrm{V}$ shows the method to estimate all the candidate routes for every flexible flight. Section VI presents the integrated system and briefs each functional block in the model. Section VII shows the simulation results of N90 metroplex and Section VIII concludes the paper.

\section{THE CONCEPTS OF METROPLEX AND FLEXIBLE FLIGHT}

A metroplex is a region with several close airports which share traffic resources such as airspace and ground transportation. More rigorously, a metroplex consists of several close airports with consequential dependencies [10]. Each metroplex subsists in a system-of-systems which includes the airports, the flights and ground traffic between them, the airline companies, Air Traffic Control (ATC) services etc. In this paper we study N90 metroplex with its three major airports serving New York City metropolitan area.

A flexible flight is routed towards a metroplex instead of a pre-determined destination airport. Unlike the regular flight having a fixed destination airport, when the flexible flight approaches the decision boundary of the destination metroplex, it will receive the instruction of which runway of which airport to land in. The flexible flight operation is based on that the passengers taking a flexible flight do not have strong preference of arriving at the specific destination airport in a metropolitan area.

The concepts of metroplex and flexible flights have two main advantages. First, it allows the air traffic control system to maximize resource utilization (runways, in this case) in an otherwise tightly constrained system. Second, it allows users to experience less delay when accessing crowded metroplex airports. We expect that the early adopters of this type of flight plan will be on-demand and general aviation users, and that the hub-and-spoke commercial air carriers will follow only if a clear benefit is very likely. There are potential advantages for all stakeholders: the FAA, the air taxi community, the general aviation community, the traditional commercial airline operators, and passengers.

To operate a flexible fight from departure to landing includes two parts. The first part is to route the flexible flight towards its destination metroplex. The second part is when the flexible flight approaches the decision boundary of the metroplex, it will be scheduled to a certain runway at one of the metroplex airports. In practice the "decision boundary" consists of a series of metering fix points [17] for the ease of operation. This paper mainly deals with the first part which is the flexible flight optimal routing algorithm while the second part scheduling will be briefly introduced.

\section{NETWORK DATA STRUCTURE FOR OPTIMAL ROUTING ALGORITHM}

The dynamic weighted shortest path algorithm is developed in this paper, which is based on the algorithm proposed by Ahuja in [18]. A network data structure is designed to generate the optimal route for each flexible flight under traffic congestion constraint. The initial network is built from navaids, fixes and waypoints provided by the Future ATM Concepts Evaluation Tool (or FACET) [19]. We build the Hash index for the network structure to dynamically maintain it.

\section{A. Build the initial network data structure}

We use the historical jet route and waypoints data to capture the real flight paths that air traffic follow and build our initial network. In order to obtain the waypoint identifiers (index) and locations (longitude and latitude) for all the waypoints, we exploit the NavigationInterface in FACET. Also using the NavigationInterface, the sequence of waypoints that defines a jet route is obtained for all jet routes.

We store the connection (edge) between every two consecutive waypoints (vertices) along each jet route. Intersection points of the jet routes are automatically considered as waypoints because an intersection point will be part of the waypoint sequence for every jet route sharing that intersection point. The edges are not directed because a jet route supports traffic in both directions (at different altitudes).

The initial network data structure is then built from the waypoints and jet routes by using boost::add_vertex and boost::add_edge functions provided by Boost $\mathrm{C}++$ Graph Library (BGL) [20]. Moreover the edge weights which will be discussed in next section are set to the map boost::weight_map(pmWeigth).

\section{B. Dynamical data structure maintenance}

We use Hash index to dynamically maintain the network. Each waypoint's latitude and longitude are changed to their absolute values and then rounded into integers. Then the positive integers are stored in a Hash key pair (lat, lon).

As the waypoints information provided by FACET is not comprehensive, we append new waypoints by importing historical real flight plans to complete the network structure. 
At the same time, all the airports are imported by treating them as waypoints too. The additional edges are obtained from the historic flight track files. Together all the additional inserted elements with the initial network formulate the final data structure to implement our routing algorithm, which is visualized in Figure. 1 .

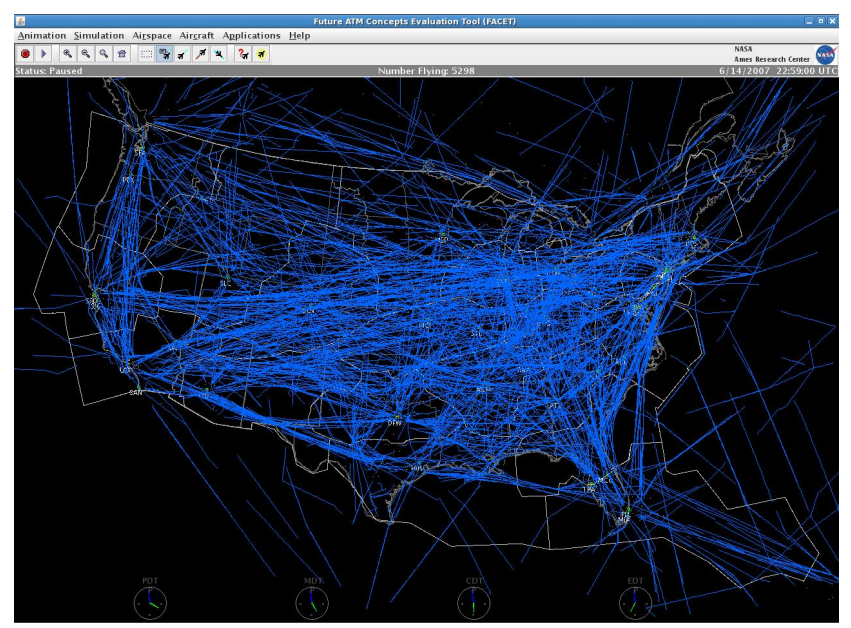

Fig. 1: The final network data structure displayed in FACET.

\section{OPTIMAL ROUTING ALGORITHMS}

In this section, we first present our static routing scheme for great circle distance weighted network and briefly introduce the mechanism and interfaces of the McTMA. Then the en route congestion constraint is taken into account and the dynamic shortest path search is adopted to find the optimal route in a time-variant weighted network structure. In the research project, we name the dynamic shortest path algorithm as the Linear Time-Variant Optimization algorithm (LTV).

\section{A. Optimal routing without dynamical congestion informa- tion}

The network data structure constructed in Section III is used for performing the optimal routing algorithm. When the routing algorithm does not take the dynamical traffic congestion into account, the weight of each edge is the great circle distance between two waypoints which is time-invariant. The Dijkstra algorithm [21] is used to find the optimal route from origin to destination. Since we are looking for the optimal route for flexible flights, the destination airport of each flight is undetermined and needs to be selected from the airports inside metroplex. In this research, the central airport of a metroplex is chosen as the destination of a flexible flight at routing phase.

Since we treat the metroplex as a super airport, we first calculate the central point $c$ of all the airports in the metroplex and then set the closest airport to $c$ as the "central" airport of the whole metroplex. The central airport will represent the destination metroplex when the flexible flights are routed to this metroplex. For example, the N90 metroplex has three airports, JFK, LGA, EWR. Firstly the geographical center is found by $c=\left(a p_{1}+a p_{2}+a p_{3}\right) / 3$, where $c$ and $a p_{i}$ are both latitude-longitude pairs. Then the closest airport LGA to the central point $c$ is selected as the "central" airport of N90.

\section{B. Airport/runway balancer and McTMA scheduler}

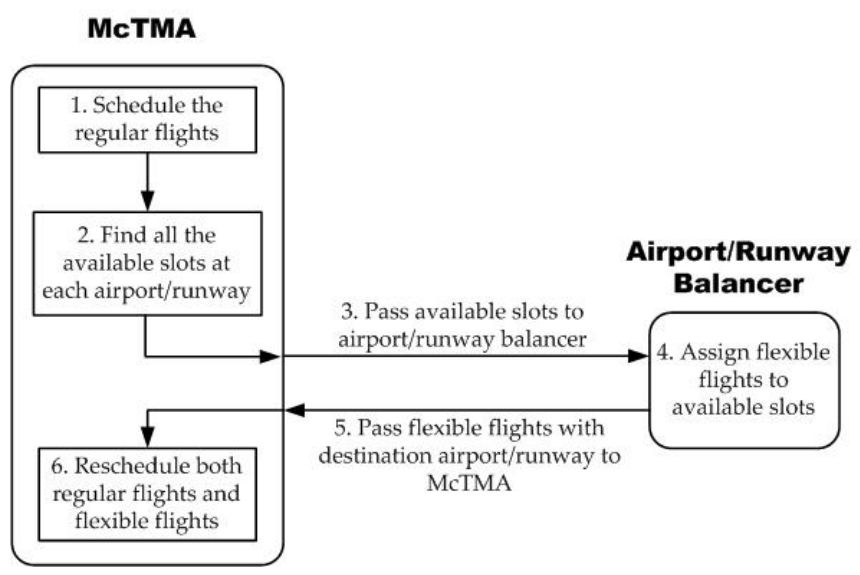

Fig. 2: Interactions between McTMA and airport/runway balancer.

In this research our algorithm generates the optimized route for a flexible flight while another team in the same project works on the runway scheduling based on McTMA. The airport/runway balancer is designed particularly for the metroplex destination to facilitate McTMA with flexible flights scheduling. The idea of their algorithm is to consider all the runways resource from all the airports in the same metroplex together. Since the regular destinationfixed flights already take most of the runway time slots, the airport/runway balancer checks the available time slots (holes) of all the runways for flexible flights. Once there is a slot available in any one or more runways of a metroplex airport, the approaching flexible flight will be scheduled to the closest runway. The airport/runway balancer can access McTMA through the interfaces shown in Figure. 2 Figure. 2 also shows the six steps of a complete metroplex runway scheduling.

\section{LTV - the Linear Time-Variant optimization algorithm}

To introduce the timely change en route traffic congestion constraint, the edges that intersect those congested areas will be assigned with a dynamic penalty weight. For advanced multiple-level congestion, the congestion impact is taken into account by assigning the edges with different congestion statuses. More congested is an area, the heavier weight it is on each intersecting edge. The dynamic congestion penalty weight will be summed up with the static great circle distance weight for each edge.

The congestion status can be forecast and published by FAA or other professional agencies. The congestion status report imported into the network data structure is used to update the edge weights and the metroplex routing is calculated based on the dynamic weights. 
In order to combine the great circle distance weight $w_{d}$ (time-invariant) and the congestion penalty weight $w_{p}$ (timevariant), we compute their normalized sum as:

$$
w(t)=w_{d}+\gamma \cdot w_{p}(t)
$$

where the larger coefficient $\gamma$ places more emphasis on congestion avoidance in the algorithm.

Therefore our task is that, given a time-variant weighted graph $G=(N, E)$ with $|N|$ nodes and $|E|$ edges, computing the shortest path for each flight under dynamical congestion condition. The weight $w_{i j}(t)$ of edge $(i, j)$ is described in Eqn. (1).

Let $w_{i j}^{*}$ denote the minimum possible weight on edge $(i, j)$, i.e., $w_{i j}^{*}=\min \left\{w_{i j}(t), \forall t\right\}$. Let $e_{i j}(t)=w_{i j}(t)-w_{i j}^{*}$ denote the excess cost for selecting edge $(i, j)$ into the route at time $t$. Let $e^{*}$ denote the maximal excess cost on any edge, i.e., $e^{*}=\max \left\{w_{i j}(t)-w_{i j}^{*}, \forall(i, j) \in E\right.$ and $\left.\forall t\right\}$.

Finally, for each $(i, j)$ and $t$, we define the cost of selecting $(i, j)$ into the route as:

$$
\begin{aligned}
c_{i j}(t) & =\alpha w_{i j}^{*}+\beta e_{i j}(t)=\alpha w_{i j}(t)+(\beta-\alpha) e_{i j}(t) \\
& =\beta w_{i j}(t)+(\alpha-\beta) w_{i j}^{*} .
\end{aligned}
$$

Suppose we solve the static shortest path problem in $G$ with the fixed weights $w_{i j}^{*}$. Let $P(k)$ denote the shortest path from node $s$ to node $k$ and $\pi(k)$ denote its travel cost. It follows from the shortest path optimality conditions [22] that:

$$
\begin{aligned}
\pi(j) \leq \pi(i)+w_{i j}^{*}, & \text { for each }(i, j) \in A, \text { and } \\
\pi(j)=\pi(i)+w_{i j}^{*}, & \text { for each }(i, j) \in P(k), \\
& \text { and each node } k \in N \backslash\{s\}
\end{aligned}
$$

By definition, $\pi(s)=0$. We define the reduced minimal travel cost $w_{i j}^{* \pi}$ as $w_{i j}^{* \pi}=w_{i j}^{*}+\pi(i)-\pi(j)$. We denote the reduced travel cost of edge $(i, j)$ as $w_{i j}^{\pi}(t)=w_{i j}(t)+$ $\pi(i)-\pi(j)$. Accordingly, $c_{i j}^{\pi}(t)=\alpha w_{i j}^{*}+\beta e_{i j}(t)$ denotes the reduced cost of $(i, j)$ at time $t$.

From the algorithm for the minimum cost walk problem in [18], we apply Algorithm 11 in following to find the dynamical weighted shortest path:

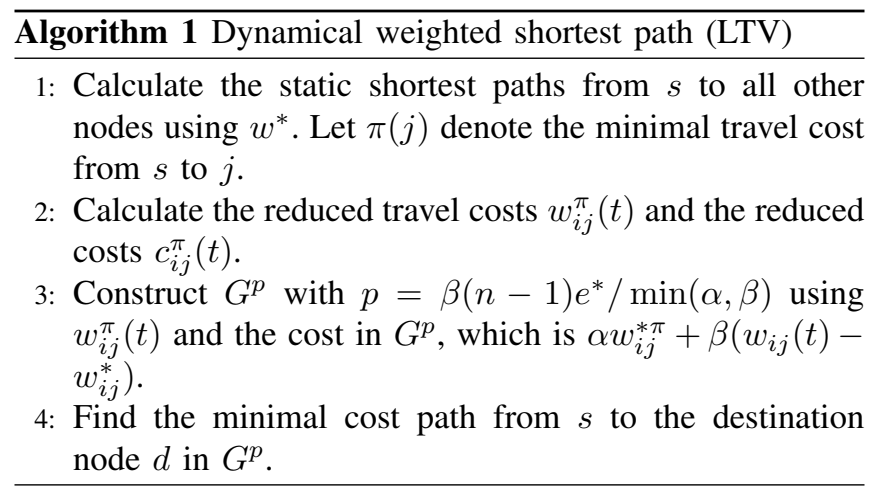

\section{CANDidate Flight Plan ESTIMATOR}

There are no flexible flight operations existed in the historical data. As a result, for each of the designated flexible flights, there is only one flight plan in the historical data whose destination is a fixed airport. To evaluate all the candidate destination airports inside the metroplex, the McTMA needs to know all possible flight plans. We find a way to estimate all the candidate flight plans from the existed flight plan for each flexible flight.

For different kinds of data, we design different estimation strategies. Given the historical data for the $i$-th flexible flight from ORD (Chicago O'hare International Airport) to JFK, whose model type is B747 and the weight category is $\mathrm{H}$, we show the estimation methods for its flexible flight route from ORD to EWR. The similar method will be applied for the estimation from ORD to LGA.

- Case 1. Same Aircraft Type

We search for B747 from ORD to EWR inside the historical flight plans. In following figure, the route in black is the original route to JFK, the route in maroon is another B747's route we found to EWR and $w p_{\text {com }}$ is the last common waypoint of the two routes. The waypoints named 40001 and 40002 are the arrival fixes for JFK and EWR respectively.

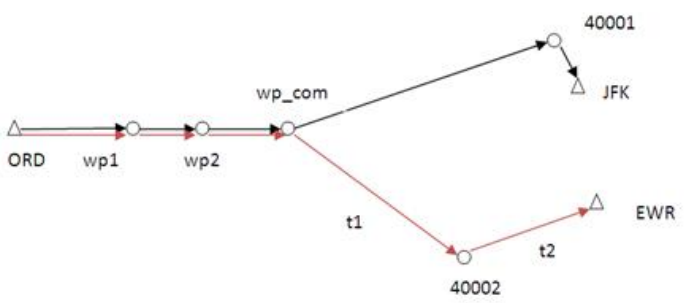

Fig. 3: The same aircraft type is found from ORD to EWR.

In this case we adopt the maroon route as our candidate and the corresponding ETAs' estimation will be:

$$
\begin{aligned}
& E T A_{40002}=E T A_{w p_{c o m}}+t_{1} \\
& E T A_{E W R}=E T A_{w p_{c o m}}+t_{1}+t 2
\end{aligned}
$$

- Case 2. Same Weight Category

If no B747 (same aircraft type) found, we search for the flight with similar type (i.e. the same weight category H). The other parts are the same as Case 1.

- Case 3. No same type or same weight category Sometimes there is only one daily flight from a small airport, e.g. LAF (Lafayette/Purdue University Airport) to JFK. We assume this flight's candidate route will follow the original route until the last common waypoint $w p_{\text {com }}$ and then head directly to arrival fix 40002 then EWR as the orange route shown in 4

To estimate the ETAs of EWR and its arrival fix 40002, 
we use the ratio of the distance method as:

$$
\begin{aligned}
& \frac{E T A_{40002}-E T A_{w p_{c o m}}}{d_{3}}=\frac{E T A_{40001}-E T A_{w p_{c o m}}}{d_{1}}, \\
& \frac{E T A_{E W R}-E T A_{w p_{c o m}}}{d_{3}+d_{4}}=\frac{E T A_{J F K}-E T A_{w p_{c o m}}}{d_{1}+d_{2}} .
\end{aligned}
$$

Then we have $E T A_{40002}$ and $E T A_{E W R}$ and the complete flight route.

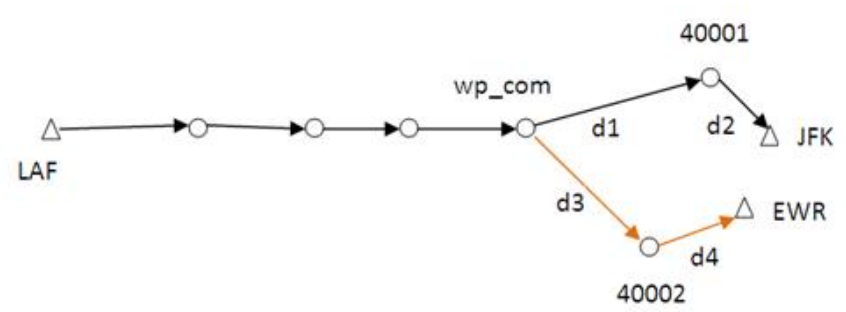

Fig. 4: No same aircraft type or same weight category is found in historical data.

In summary the methods in the three cases above are the key rules for the Candidate Flight Plan Estimator, which is another important functional block in our integrated model.

\section{INTEGRATED MODEL}

In order to evaluate the optimal routing algorithm and the McTMA scheduling result for flexible flight operations, we create an integrated model which consists of several functional blocks. We also illustrate how these blocks are connected and the data flow diagram in Figure 5

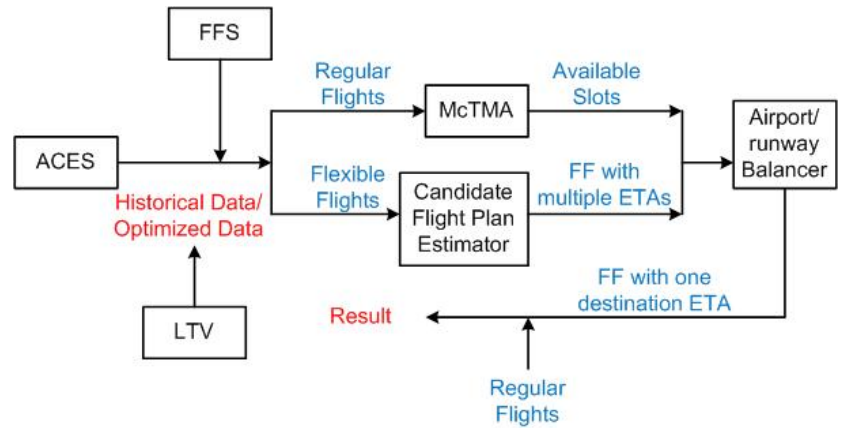

Fig. 5: The data flow diagram of the integrated model.

Besides LTV, there are five major functional blocks in Figure 5. They are ACES, FFS, Candidate Flight Plan Estimator, McTMA scheduler and the airport/runway balancer.

ACES (Airspace Concept Evaluation System) [23] processes the historical flight plan data and outputs the data with Airline ID, computer ID, aircraft type, weight category, origin airport, destination airport, the sequence of waypoints and the ETAs, altitude, airspeed along the trajectory. The resulted flight plans are separated by FFS into regular flights and flexible flights.

FFS (Flexible Flight Selection) takes the same set of historical data from ACES and designates the flexible flights based on the probability of low connecting passenger numbers. Thus it separates the data into regular flights and flexible flights and feed the data to McTMA and Candidate Flight Plan Estimator. This part is developed by another team in the same project.

The historical flight plans of flexible flights are sent to Candidate Flight Plan Estimator. Based on one existed flight plan, several new candidate flight plans are estimated, which are designed for the same flexible flight with different destination airports inside the metroplex.

McTMA [16] takes the regular flights and runs the scheduler to find out the available time slots (holes) for the flexible flights. These holes are then sent to the airport/runway balancer that will decide which destination airport a flexible flight should land in. Now every flexible flight only has one destination airport and one flight plan. Then the selected flexible flight plans go back into McTMA and McTMA will do the second time scheduling. The second time scheduled routes and ETAs are the final output of the entire system and the results will be shown and evaluated in next section.

The airport/runway balancer accepts all the candidate flight plans for each flexible flight from the Candidate Flight Plan Estimator. One of these flight plans is selected and all the selected flight plans are sent back to McTMA where they are scheduled together with all the regular flight plans.

\section{SimULATION}

The simulation was performed on the historical data of November 7, 2008 at 3 major airports of N90.

Before testing the flexible flight operation concept, we attempted to identify the benefit of the routing optimization performed by the LTV algorithm when there were no flexible flights. Figure 6 shows the comparison of total delay for the nominal flights in baseline historical data with the LTVoptimized results. With the help of LTV, the total delay at the three airports was reduced to 1,830 minutes (9.5\% reduction) as compared to the 2,025 minutes for the baseline traffic file.

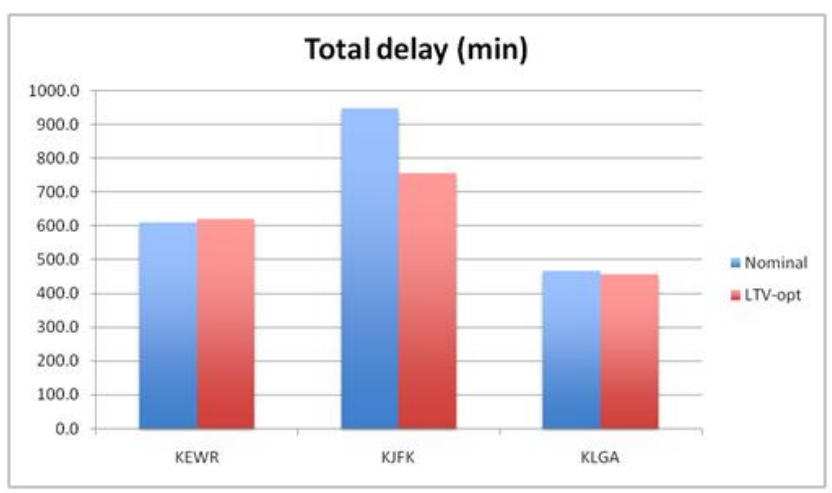

Fig. 6: The effect of LTV at three major airports in N90 on November 7th, 2008.

More detailed delay statistics for both nominal and LTVoptimized flights are shown in Table I Overall, the LTV optimizer has a positive effect on metroplex total delay reduction.

A comparison of the total delay for the four different approaches is shown in Figure 7. Without Flexible Flight 
Operations (FFO), the LTV makes relatively small delay reduction by optimizing the en route traffic (about 10\%). Without the LTV, applying FFO to $30 \%$ of the flights in historical data $30 \%$ are flexible flights, $70 \%$ are regular flights) gives about 300 minutes total delay reduction because the FFO well balanced the runway resource and provided McTMA scheduler with better ETAs. In summary, combining both LTV optimization and flexible flight operations has the largest delay reduction which is also the final result of our integrated model.

TABLE I: Statistics of N90 traffic before and after LTV optimization on November 7th, 2008.

\begin{tabular}{l|cc|cc|cc}
\hline \multirow{2}{*}{ Delay on N90 } & \multicolumn{2}{|c|}{ EWR } & \multicolumn{2}{|c|}{ JFK } & \multicolumn{2}{c}{ LGA } \\
& non-opt & opt & non-opt & opt & non-opt & opt \\
\hline mean & 1.15 & 1.17 & 1.96 & 1.56 & 0.91 & 0.89 \\
median & 0.33 & 0.55 & 0.95 & 0.74 & 0.40 & 0.38 \\
SD & 1.71 & 1.49 & 2.70 & 2.18 & 1.09 & 1.09 \\
max & 9.70 & 9.13 & 12.88 & 10.28 & 4.88 & 5.85 \\
3rd quartile & 1.65 & 1.90 & 2.85 & 2.26 & 1.53 & 1.53 \\
\hline
\end{tabular}

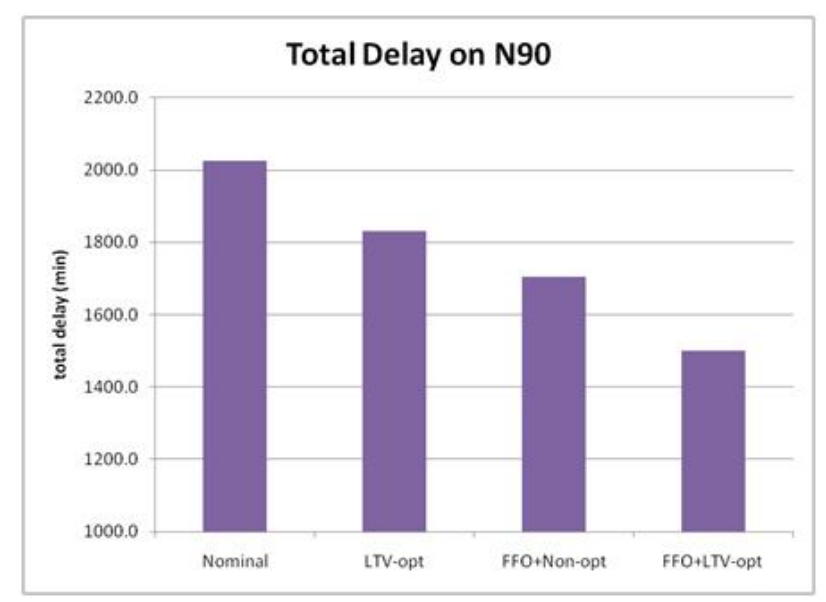

Fig. 7: Total delay comparison for the four approaches for the November 7th, 2008 traffic.

\section{CONCLUSION}

In this work the concepts of metroplex and flexible flights are introduced. To reduce the total delay of the metroplex airports, we design the optimal flexible routing algorithm based on a time-variant weighted network structure, in which the en route traffic congestion is dynamically changed. The McTMA scheduling scheme and its interfaces are also demonstrated. We present the integrated model to support the optimal metroplex routing, scheduling and flexible flight operations. The numerical result confirms the benefits of both the routing algorithm and the entire system.

\section{ACKNOWLEDGEMENTS}

The authors acknowledge the sponsorship of this research by the National Aeronautics and Space Administration under contract NNL10AA15C.

\section{REFERENCES}

[1] F. A. Administration, "FAA Aerospace Forecasts FY 2008-2025," December 2010.

[2] A. Donaldson and R. Hansman, "Capacity improvement potential for the new york metroplex system," in 10th AIAA Aviation Technology, Integration and Operations (ATIO) Conference and AIAA/ISSMO Multidisciplinary Analysis Optimization (MAO), Fort Worth, TX, Sep 2010.

[3] S. Atkins, "Investigating the nature of and methods for managing metroplex operations: Initial site survey report (NCT)," NASA Metroplex NRA Project Report, Contract No.NNA07BC56C.

[4] _ - "Investigating the nature of and methods for managing metroplex operations: New york metroplex site visit report," NASA Metroplex NRA Project Report, Contract No.NNA07BC56C.

[5] S. Atkins and S. Engelland, "Observation and measurement of metroplex phenomena," in Digital Avionics System Conference, October 2008.

[6] L. Wang, G. Donohue, K. Hoffman, L. Sherry, and R. Oseguera-Lohr, "Analysis of air transportation for the new york metroplex: Summer 2007," in Proceedings International Conference on Research in Air Transportation, Fairfax, VA, Febuary 2008.

[7] G. Donohue, K. Hoffman, L. Wang, and D. DeLaurentis, "Metroplex operations: Case study of new york metroplex air transportation system," in NASA Airspace Systems Program, Austin, TX, March 2008.

[8] D. Schaar, L. Sherry, and G. L. Donohue, "Rethinking airport improvement: Analysis of domestic airline service to u.s. metroplex airports," in CASTR Internal Report CASTR-01-2010, March 2010.

[9] E. McClain and J. P. Clarke, "Traffic volume intersection metric for metroplex clustering analysis," in AIAA Guidance, Navigation, and Control Conference, Aug 2009.

[10] D. DeLaurentis and S. Ayyalasomayajula, "Analysis of dependencies and impacts of metroplex operations," October 2010, purdue University.

[11] J. Krozel, J. Prete, J. Mitchell, P. Smith, and A. Andre, "Designing on-demand coded departure reroutes," in AIAA Guidance Navigation and Control Conference and Exhibit, Keystone, CO, August 2006.

[12] J. Krozel, S. Penny, J. Prete, and J. Mitchell, "Automated route generation for avoiding deterministic weather in transition airspace," Journal of Guidance, Control, and Dynamics, vol. 30, no. 1, pp. 144153, January 2007.

[13] A. Mukherjee and M. Hansen, "A dynamic rerouting model for air traffic flow management," Transportation Research Part B, vol. 43, pp. 159-171, 2009.

[14] C. Taylor and C. Wanke, "Dynamic generation of operationally acceptable reroutes," in 9th AIAA Aviation Technology, Integration, and Operations Conference (ATIO), Hilton Head, South Carolina, September 2009.

[15] B. Capozzi, S. Atkins, and S. Choi, "Towards optimal routing and scheduling of metroplex operations," in 9th AIAA Aviation Technology, Integration, and Operations Conference (ATIO), Hilton Head, South Carolina, September 2009.

[16] S. Landry, "The design of a distributed scheduling system for multicenter time-based metering of air traffic into congested resources," Air Traffic Control Quarterly, vol. 16(1), pp. 69-97, 2008.

[17] H. Idris, A. Evans, and S. Evans, "Single-year nas-wide benefits assessment of multi-center tma," 2004, titan Corporation Air Traffic Systems Division.

[18] R. Ahuja, S. Pallottino, and M. Scutella, "Dynamic shortest paths minimizing travel times and costs," Networks, vol. 41, p. 197C205, 2003.

[19] K. Bilimoria and B. Sridhar, "Facet: Future atm concepts evaluation tool," in 3rd USA/Europe Air Traffic Management R\&D Seminar, Napoli, Italy, 2000.

[20] J. G. Siek, L.-Q. Lee, and A. Lumsdaine, Boost Graph Library, The User Guide and Reference Manual, 1st ed. Addison-Wesley Professional, Dec 202001.

[21] E.W.Dijkstra, "A note on two problems in connexion with graphs," Numerische Mathematik, vol. 1, pp. 269-271, 1959.

[22] R.K.Ahuja, T. Magnanti, and J. Orlin, Network flows: Theory, Algorithms, and Applications. NJ: Prentice Hall, 1993.

[23] S. George, G. Satapathy, and V. Manikonda, "Build 8.0 of the airspace concept evaluation system," in AIAA Guidance, Navigation, and Control Conference, Portland, OR, Aug 2011. 